\title{
Narrative event boundaries, reading times, and expectation
}

\author{
Kyle A. Pettijohn ${ }^{1} \cdot$ Gabriel A. Radvansky ${ }^{1}$
}

Published online: 11 May 2016

(C) Psychonomic Society, Inc. 2016

\begin{abstract}
During text comprehension, readers create mental representations of the described events, called situation models. When new information is encountered, these models must be updated or new ones created. Consistent with the event indexing model, previous studies have shown that when readers encounter an event shift, reading times often increase. However, such increases are not consistently observed. This paper addresses this inconsistency by examining the extent to which reading-time differences observed at event shifts reflect an unexpectedness in the narrative rather than processes involved in model updating. In two reassessments of prior work, event shifts known to increase reading time were rated as less expected, and expectedness ratings significantly predicted reading time. In three new experiments, participants read stories in which an event shift was or was not foreshadowed, thereby influencing expectedness of the shift. Experiment 1 revealed that readers do not expect event shifts, but foreshadowing eliminates this. Experiment 2 showed that foreshadowing does not affect identification of event shifts. Finally, Experiment 3 found that, although reading times increased when an event shift was not foreshadowed, they were not different from controls when it was. Moreover, responses to memory probes were slower following an event shift regardless of foreshadowing, suggesting that situation model updating had taken place. Overall, the results support the idea that previously observed reading time increases at event shifts reflect, at least in part, a reader's unexpected encounter with a
\end{abstract}

Kyle A. Pettijohn

Pettijohn.1@nd.edu

1 Department of Psychology, University of Notre Dame, 260 Haggar Hall, Notre Dame, IN 46556, USA shift rather than an increase in processing effort required to update a situation model.

Keywords Situation models $\cdot$ Memory $\cdot$ Reading

Events that people read about are often changing, and people's comprehension and understanding of them need to be properly represented and updated. The referential level of representation that captures the described events is the situation model level (Johnson-Laird, 1983; van Dijk \& Kintsch, 1983; Zwaan \& Radvansky, 1998). Frequently, the reader's situation model needs to be updated to incorporate new information, or a new model needs to be created to represent a new event. The event indexing model (Zwaan, Langston, \& Graesser, 1995) identifies a number of dimensions along which a situation can change. Changes along these dimensions can be thought of as event shifts. A common finding in the literature is that reading times often increase when an event shift is encountered (e.g., Radvansky \& Copeland, 2010; Rinck \& Weber, 2003; Zwaan, Magliano, \& Graesser, 1995; Zwaan, Radvansky, Hilliard, \& Curiel, 1998). The aim of this paper is to explore the role of causal unexpectedness as a contributor to the processing of these event shifts. That is, are the increased reading times observed at event shifts only because of the additional processing effort required to update a situation model, do they reflect the unexpectedness of a causal break in which the reader may be uncertain why an event shift has suddenly occurred in the text, or is it a combination of processing effort and unexpectedness?

\section{Situation model processing during reading}

The event indexing model (Zwaan, Magliano, et al., 1995; Zwaan, Langston,et al., 1995; Zwaan \& Radvansky, 1998) 
suggests that people track several different event indices as they read a text. Typically, five indices are identified: space, time, protagonist (or entity), intentionality (goals), and causality (see also Kurby \& Zacks, 2012; McNerney, Goodwin, \& Radvansky, 2011; Speer, Zacks, \& Reynolds, 2007). By tracking these event dimensions, people can identify event shifts, which could correspond to changes along any of these dimensions. For example, when reading a story in which a character goes from her home to the museum, the change in spatial location is a signal that a new event has occurred, and readers would need to update their situation model to take into account this new event.

There is a great deal of evidence that reading times increase when people encounter event shifts. However, this not universally or consistently observed. Research findings for each of the typically assessed event dimensions are presented in Table 1. Overall, although there is consistent evidence that causal shifts result in increased reading times, the evidence for the other event dimensions is less consistent. We consider each of them in turn.

Spatial shifts occur when the narrative action moves from one location to another. For example, if a character is watching television at home and then meets some friends at a pub, a spatial shift has occurred. Some studies have found that spatial shifts lead to increased reading times (Radvansky, Zwaan, Curiel, \& Copeland, 2001; Zwaan, Magliano, et al., 1995; Zwaan et al., 1998). However, other studies have found no difference (Radvansky \& Copeland, 2010; Zwaan, Magliano, et al., 1995; Zwaan et al., 1998) or only a marginal reading time increase (Rinck \& Weber, 2011). One study found a significant reading-time decrease (McNerney et al., 2011).

Temporal shifts occur when there is a sizeable gap in narrative time. The length of time needed to convey a shift may vary, depending on the context. For example, "five hours later" signals a new event when reading about a person watching a movie, but "20 minutes later" generally does not (Anderson, Garrod, \& Sanford, 1983). When a new time frame has been identified, because readers must establish a new temporal framework, a new situation model may be created. Several studies have found increased reading times accompanying temporal shifts (Radvansky \& Copeland, 2010; Radvansky et al., 2001; Rinck \& Weber, 2003; Zwaan, Magliano, et al., 1995; Zwaan et al., 1998). However, other studies have found decreases in reading times for historical texts (Radvansky et al., 2001) and in a novel (McNerney et al., 2011).

Goal or intention changes occur when a story's protagonist has a new goal to achieve. For example, if a story states that Jimmy wants a new bike, this is a new goal for the character and sets up a new series of events. When a protagonist's intention or goal changes, there is generally an increase in reading time (Radvansky et al., 2001; Zwaan et al., 1998), although sometimes the effect is only marginal (Zwaan et al., 1998) or not significant (McNerney et al., 2011).

Entity shifts occur when either the protagonist of the story changes or a new character is introduced. For example, when a story has been centered on John and the narrator begins describing events involving Julia. Generally, shifts along this dimension result in an increase in reading time (McNerney et al., 2011; Rinck \& Weber, 2003; Zwaan et al., 1998), although not exclusively. In at least one case, it led to significantly faster reading times (Radvansky et al., 2001, Exp. 1) for narratives written as historical texts.

Causal shifts occur when activity in a narrative cannot be explained by the prior story events. For example, if a character is drumming and suddenly becomes angry, it would serve as a

Table 1 Effect of situation model variable on reading time

\begin{tabular}{|c|c|c|c|c|c|c|c|}
\hline \multirow[t]{2}{*}{ Authors } & \multirow[t]{2}{*}{ Year } & \multirow[t]{2}{*}{ Experiment } & \multicolumn{5}{|c|}{ Situation Model Variable } \\
\hline & & & Spatial & Temporal & Goal & Entity & Causal \\
\hline \multirow[t]{2}{*}{ Zwaan, Magliano, \& Graesser } & \multirow[t]{2}{*}{1995} & 1 & $n s$ & + & & & + \\
\hline & & 2 & + & + & & & + \\
\hline \multirow{3}{*}{$\begin{array}{l}\text { Zwaan, Radvansky, Hilliard, } \\
\text { \& Curiel }\end{array}$} & \multirow[t]{3}{*}{1998} & 1 & $n s$ & + & $+\%$ & + & + \\
\hline & & 2 & + & + & + & + & + \\
\hline & & 3 & $n s$ & + & + & + & + \\
\hline \multirow{2}{*}{$\begin{array}{l}\text { Radvansky, Zwaan, Curiel, } \\
\text { \& Copeland }\end{array}$} & \multirow[t]{2}{*}{2001} & 1 & $+*$ & - & + & - & $+* *$ \\
\hline & & 2 & + & + & + & $t^{*}$ & + \\
\hline Rinck \& Weber & 2003 & $1 \& 2$ & $+\%$ & + & & + & \\
\hline \multirow[t]{2}{*}{ Radvansky \& Copeland } & \multirow[t]{2}{*}{2010} & 1 & $n s$ & & & & \\
\hline & & 2 & & + & & & \\
\hline $\begin{array}{l}\text { McNerney, Goodwin, } \\
\text { \& Radvansky }\end{array}$ & 2011 & & - & - & $n s$ & + & + \\
\hline
\end{tabular}

Note. + Indicates increased time, - indicates decreased time, ns indicates not significant, blank space indicates the variable was not tested. \% - Marginal; * Significant in older subjects only; ** Significant in younger subjects only 
causal shift because it is not clear why such anger would happen (Graesser, Singer, \& Trabasso, 1994). Readers link subsequent events back to the causal shift to either explain why it happened or to provide a motivation for or explanation of the events that followed (e.g., perhaps it is revealed that the drummer had broken a new drumhead). Previous work suggests that causal shifts reliably lead to increased reading times (McNerney et al., 2011; Radvansky et al., 2001; Zwaan, Magliano, et al., 1995; Zwaan et al., 1998). Causality is the only dimension that seems to consistently influence reading time, and in the same way (i.e., reading times always increase).

\section{Situation model updating and expectation}

Consistent with the idea that causality plays an overarching role in influencing understanding, some researchers (e.g., Long \& Lea, 2005) have proposed that as readers comprehend a text, they maintain coherence through a passive resonance process. This process activates real-world and prior-text information that is then used to interpret new information as it is encountered. Even when a related concept is not necessary for understanding the text, it will be activated (O'Brien \& Albrecht, 1991). When a sentence appears that contradicts something presented several sentences earlier, readers will note the inconsistency even though the information should be backgrounded and even though local coherence is maintained (Albrecht \& O'Brien, 1993). Additionally, reading times are slower for sentences that contain the contradiction (Lea, Kayser, Mulligan, \& Myers, 2002). Thus, when expectations are subverted, it can affect the amount of time needed to understand a sentence. In other cases, when this passive process is not enough for full comprehension, readers may use information from schemata, scripts, or other sources to make inferences about the text (Lea et al., 2002). When these processes fail, or when readers need to make inferences, it may be so unexpected as to create a causal shift which then reduces the causal connectedness of the narrative. When model updating occurs, the new situation is stable, and connectedness is reestablished. The extra processing associated with updating should be reflected in increased reading times.

Related to the idea that causality is key to understanding a narrative, event segmentation theory (EST) (Zacks, Speer, Swallow, Braver, \& Reynolds, 2007) stated that people are constantly trying to predict the future state of an event (Kurby \& Zacks, 2008). When the discrepancy between the predicted and actual states is too high, an error monitoring system triggers an update to the current model or the creation of a new model. Similarly, the unexpectedness of an event shift can cause this updating by acting as a causal break. The mismatch caused by the discontinuity of the event increases the error rate, which may, in turn, lead to the need to generate inferences about what will happen next. Thus, the reader's ability to connect the relationship between the new and old events will affect prediction accuracy.

When a narrative event shift occurs, there may not be a clear reason. As such, these shifts may be unexpected by the reader and may act more like causal shifts. For example, the study that found faster reading times for spatial shifts (McNerney et al., 2011) used a real-world novel. It is possible that this made spatial shifts easier to process because the rich source of information allowed for causal connections to easily be made, thereby making them more expected. This is in comparison to most laboratory experiments that used shorter texts in which it is often difficult for readers to develop an expectancy of what will happen next. For example, it may not be clear why, in the limited text that is provided in most experimental stories, the action may be moving from one location to another. This idea that event shifts are processed more effectively when the reason for them is apparent from the text is consistent with work showing that people read sentences faster when there was a functional spatial relationship, such as standing under a bridge versus under a lamppost during a rainstorm (Radvansky \& Copeland, 2000; Radvansky, Copeland, \& Zwaan, 2003). Similarly, when people read texts in which the spatial relation between an object and its location was causally relevant to the text, they responded to both (object and location) probes faster than when the relationship was not causally relevant (Sundermeier, van den Broek, \& Zwaan, 2005).

Other studies have found that people use causal information to further their understanding of a narrative. For example, when people read sentences that described an event, causal relevance was a necessary condition for them to spontaneously generate spatial models of that event (Jahn, 2004). People also monitor model dimensions and the ways they interact to aid their comprehension. The spatial distance between a protagonist and an object in a narrative can affect how available the object is to memory (e.g., Glenberg, Meyer, \& Lindem, 1987). When time was used to implicitly vary this distance (e.g., the protagonist listened to half versus the introduction to a recorded lecture while walking), the initial location was less available the longer the activity would have taken (Rapp \& Taylor, 2004). Thus, people will generate inferences based on the relationships between event model dimensions.

This suggests that when a readily apparent causal connection exists, there is reduced need to infer a relationship and less processing is required. Because of this, it may be that the ability to account for the cause of a spatial shift (or spatial relation) influences reading times and associated processing. It is possible that other situation model shifts function in much the same way: When a shift occurs, the reader must infer a relationship between the new information and that in the current model. This disparity may function more as a causal shift, and the less expected the new information, the more one has to search for meaning and generate inferences. However, the focus of this paper is on the effect of event shifts on reading 
times and model updating, given a certain amount of information, rather than the text's coherence or the process of inference generation.

To address the issue of whether reading-time increases are caused by an increase in cognitive effort or because of unexpectedness associated with a functional causal shift, we performed a number of studies. This involved a comparison of ratings of existing materials and the reading-time data obtained with them (Radvansky et al., 2001) as well some experiments that involved new data collection with new materials. For the studies involving the Radvansky et al. (2001) materials, the first study assessed whether sentences identified as event shifts using the event indexing model were less expected compared to other sentences. The second study compared these expectancy ratings to the reading-time data gathered by Radvansky et al. (2001). Experiment 1 involved ratings of expectedness for materials that better controlled for the number and type of event shifts. Experiment 2 assessed whether foreshadowing influenced event-shift detection. Finally, Experiment 3 examined online processing of event shifts and memory for information encountered before the shift to assess cognitive effort and model updating.

\section{Study 1}

The aim of the first study was to determine if the event shifts in previously existing materials (which had shown increases in reading times) were more unexpected compared to controls in which there was no such event shift. To do this, expectedness ratings of a particular sentence were gathered for both the original sentence and an altered control version.

\section{Method}

Participants Eight hundred seventy-nine participants were recruited using Amazon's Mechanical Turk service. Participants were all over the age of 18 years and were residents of the United States. Each was paid 5 cents for completing the experiment.

Materials and procedure Eight stories from Radvansky et al. (2001) were used. These stories were 32 to 46 sentences long. Four of the stories were about historical events, such as the gunpowder plot of 1605; the other four were the same stories modified to read more like a narrative and set in the present. To evaluate the expectedness of each sentence, each person saw two five-sentence excerpts from the texts. These excerpts included a target sentence and the four sentences that preceded it. The first sentence of each story was never presented because it was assumed to have maximal unexpectedness because nothing came before it. For the second, third, and fourth sentences of the stories, excerpts contained only the first two, three, or four lines of each story, respectively. Note that the target sentences were all of the sentences from each text (excluding the first sentence), including those that did and did not convey event shifts.

To assess the unexpectedness of a target sentence, an alternate version was created that conveyed no event shift. This was accomplished by removing information that referred to a shift, such as the introduction of a new entity or a change in location. For example, a sentence that conveyed a temporal shift, such as, "In a few years, some bulbs were shipped out" was rewritten as "Some bulbs were shipped out." This process resulted in a total of 548 unique excerpts that were used for testing.

Excerpts were presented using the Qualtrics Survey Software website. After reading a brief description of the experiment and providing consent, participants read the two excerpts and rated the last sentence of each for expectedness. This was done using a scale from 1 (least expected) to 7 (most expected). Excerpts were paired randomly for a given person with the restriction that each pair contained one excerpt that ended with a sentence that conveyed a shift (or shifts) and one excerpt that ended with a no-shift sentence. All of the sentences from each excerpt were presented on the same screen, and the two excerpts appeared on different screens (i.e., not at the same time). After reading and rating, a debriefing screen was presented explaining purpose of the study, and participants were given a code to enter in Mechanical Turk for compensation. Three ratings were obtained for each sentence.

\section{Results and discussion}

The data were analyzed to address whether there is an expectedness difference for shift and nonshift sentences. For this analysis, temporal shifts were divided between absolute and relative shifts. This corresponds to a difference in a given date (e.g., in March 1605 . . .) versus a difference relative to narrative time (e.g., three months later . . .). There was no difference between the two types of temporal shifts, so they are not discussed further.

A one-way ANOVA comparing expectedness ratings between sentences that did or did not convey event shifts in the original materials showed that the shift sentences were rated as less expected than nonshift sentences $(M=4.21, S E=0.08 ; M$ $=4.82, S E=0.16$, respectively); $F(1,301)=8.85, M S E=$ 13.42, $p=.003, \eta_{\mathrm{p}}^{2}=.03$. A one-way ANOVA comparing target shift sentences and the versions that were altered such that they did not convey a shift showed that shift sentences had lower expectedness ratings than their nonshift counterparts $(M=4.34, S E=0.06 ; M=4.87, S E=0.06$, respectively); $F(1,1757)=40.52, M S E=123.00, p<.001, \eta_{\mathrm{p}}^{2}=.02 .{ }^{1}$

\footnotetext{
${ }^{1}$ For both studies and all of the experiments, differences between various shift types were also examined. The different shift types did not interact with any other variable, suggesting that participants processed them similarly. Therefore, all of our results were collapsed across shift type to simplify our exposition.
} 
The results of Study 1 suggest the presence of situation model shifts affects judgments of expectedness. Although this assessment is consistent with the idea that event boundaries all convey a disruption of the causal connections between events - because there was no experimental manipulation of shifts, and the number and types of shifts were conflated in each sentence - it is difficult to adequately assess whether the effects observed here are due to the presence of a shift or to other variables that may be present in the materials.

Another concern that might be raised about this study is that participants only saw five sentence segments of the materials, often not the entire text leading up to that point. This was done so as not to burden people with reading large amounts of material that often did not bear on the issue at hand. Given this choice we made, we acknowledge that it is possible that these results might be different if people had been allowed to read everything up to that point. However, having noted this concern, we would also point out that the pattern of results this study are in line with the other studies and experiments reported here, and so any deviation from the current results are likely to be minor.

\section{Study 2}

The aim of the second study was to determine if the expectedness rating of a particular sentence was related to reading times for that sentence in addition to other measures that are known to influence reading time, such as word frequency and the presence of event shifts. Reading times for each subject in the Radvansky et al. (2001) data set were included in multiple regression analyses along with the expectedness ratings obtained in Study 1. The reading-time data were analyzed using mixed-effects multipleregression models, with participant as a random effect. For the initial model, reading times were regressed on number of syllables, sentence position, number of new arguments, mean word frequency, and the six shift types (space, absolute time, relative time, entity, cause, and goal). Analyses were carried out using the lme4 (Bates, Mächler, Bolker, \& Walker, 2015) and lmerTest (Kuznetsova, Brockhoff, \& Christensen, 2013) packages in R (R Core Team, 2013). A second model was fitted, adding the mean expectedness rating for each sentence, the number of shifts in the sentence, and the interaction of these two factors.

\section{Results and discussion}

The $t$ values for each predictor and their associated significance values are presented in Table 2. As can be seen, the situation model variables were all significantly different from zero, with the exception of relative time. When the additional variables were added, most of the situation model variables remained significant; however, relative time became a significant predictor, and goal was not. The expectedness rating of each sentence, number of shifts, and the interaction of these two variables were
Table 2 Model results for Study 2

\begin{tabular}{|c|c|c|c|c|}
\hline & \multicolumn{4}{|c|}{ Expectedness Rating } \\
\hline & \multicolumn{2}{|c|}{ Not included } & \multicolumn{2}{|c|}{ Included } \\
\hline & $t$ & $p$ & $t$ & $p$ \\
\hline \multicolumn{5}{|l|}{ Auxiliary variables } \\
\hline Syllables & 80.77 & $<.001$ & 75.51 & $<.001$ \\
\hline Position & -4.99 & $<.001$ & -4.40 & $<.001$ \\
\hline New arguments & 8.02 & $<.001$ & 7.98 & $<.001$ \\
\hline Average frequency & 1.75 & 0.081 & 2.09 & 0.037 \\
\hline Expectedness & & & -6.86 & $<.001$ \\
\hline Number of shifts & & & -2.00 & 0.046 \\
\hline Expectedness $\times$ number of shifts & & & 5.71 & $<.001$ \\
\hline \multicolumn{5}{|l|}{ Situation model variables } \\
\hline Space & -4.58 & $<.001$ & -4.93 & $<.001$ \\
\hline Absolute time & 5.06 & $<.001$ & 1.98 & 0.048 \\
\hline Relative time & -1.17 & 0.241 & -2.17 & 0.030 \\
\hline Entity & 10.75 & $<.001$ & 6.36 & $<.001$ \\
\hline Cause & 6.14 & $<.001$ & 2.19 & 0.029 \\
\hline Goal & 2.78 & 0.005 & 0.61 & 0.540 \\
\hline
\end{tabular}

significant; note that because the scale here runs from least to most expected, the negative coefficient indicates that reading times decreased as expectedness increased. The additional predictors significantly improved the model's goodness of fit, $\chi^{2}=$ $63.72, p<.001$, suggesting that the more complicated model should be retained. To explore the nature of the interaction between expectedness rating and number of shifts, an additional model was fitted using sentences with an average expectedness rating of six or above (10.67\% of the sentences) in addition to the auxiliary variables. The result was that the number of shifts was no longer a significant predictor of reading times, $p=.25$, which suggests that, when a sentence is more expected, reading times are less affected by event shifts. This topic is explored in more depth in Experiment 2.

The results of Study 2 suggest that reading times increase in response to shifts along several situation model dimensions as well as to sentences that contain information that is not expected. Thus, it may be that when people do not expect information, they have a harder time making causal connections between the new and old material, and this difficulty is reflected in increased reading times. Study 2 is subject to the same limitations as Study 1 with respect to experimental manipulations. In addition, the materials were not designed to elicit differences in unexpectedness. As such, they conflate the type and number of shifts present in each sentence, which may have affected expectedness ratings. The following experiments were designed to address these limitations.

\section{Experiment 1}

The aim of Experiment 1 was to determine if people find sentences containing an event shift more or less expected than 
those that do not and to assess how foreshadowing impacts expectedness. If people find sentences that convey an event shift less expected, it would support the idea that these sentences may represent a causal break to the reader. Stories were presented that contained an event shift, and it was either foreshadowed or not.

\section{Method}

Participants One hundred thirty people (69 female) were recruited through Amazon's Mechanical Turk website. Participants ranged in age from 18 to 77 years $(M=36.34$, $S D=12.68)$. Participants who failed to correctly answer 12 of 16 comprehension questions (75\%) were excluded. This resulted in the elimination of 18 participants. Participants were paid for their participation.

Materials Forty-eight stories between six and seven sentences in length were used (an example of one of the stories used is included in the Appendix). Each story had four versions that differed in terms of whether a particular event shift occurred, and if so, if it was foreshadowed. Foreshadow sentences were written to create an expectation that the situation might change but not explicitly state what the change would be. For example, the foreshadow sentence in the story that is included in the Appendix states that the character would need to go to bed soon. This indicates that a change may be imminent but not what it will be. It is conceivable that the event shift could be in spatial location, goal, or (as is the case in this example) time. The possible versions of each story were No Foreshadow No Shift, No Foreshadow Shift, Foreshadow No Shift, and Foreshadow Shift (see Appendix). Participants saw eight experimental and eight filler stories; stories were randomized and counterbalanced. Only one event shift occurred in each target sentence. There were eight stories for each of five shift types, plus the additional eight filler stories. Causal shifts were included as a baseline comparison of the influence of a causal shift on processing. Because, by definition, a foreshadowed causal shift is not a causal shift, these stories were analyzed separately. After each story, participants were asked to answer a single true/false comprehension question to ensure that they were actually reading the materials. These comprehension questions pertained to nonshift story information.

Procedure Participants read eight experimental and eight filler stories. After reading a brief description of the experiment and providing informed consent, they read instructions explaining how to progress through the experiment. Participants were told to press the space bar to advance to the next sentence of each story. Sentences were presented one at a time, and participants rated the expectedness of each sentence based on the one that immediately preceded it. Ratings were made on a scale of 1 (completely expected) to
7 (completely unexpected). Experimental story versions were presented in one of four subsets that were randomized with the restriction that the first story was a filler story to familiarize participants with the task.

Participants were also asked to provide demographic information and information regarding the testing environment at the end of the experiment. Demographic information included participant's gender, age, highest education level attained, ethnicity, handedness, and whether the person was a native English speaker. Environmental information included noise level, type of device used to respond, type of Internet connection, and alertness level. Data from two subjects who indicated they were not native English speakers or who completed the task on a smart phone or tablet were replaced. Finally, participants read a debriefing screen and received a code for compensation.

\section{Results and discussion}

Because the main concern in Experiments 1 and 2 is the effect of foreshadowing on the dependent variables, ratings were collapsed across stories, and the analyses were performed at the item level. The ratings for each condition are reported in Table 3. The data were submitted to a 2 (foreshadow) $\times 2$ (shift) repeated-measures ANOVA.

For the noncausal shift stories, the main effects of foreshadow, $F(1,31)=6.35, M S E=0.901, p=.017, \eta_{\mathrm{p}}^{2}=0.17$, and shift, $F(1,31)=15.18, M S E=1.28, p<.001, \eta_{\mathrm{p}}^{2}=0.33$, were significant. Importantly, the interaction was significant, $F(1$, 31) $=12.43, M S E=0.678, p=.001, \eta_{\mathrm{p}}^{2}=0.29$. Simple effects tests revealed that for the no-foreshadow condition, the effect of shift was significant, $F(1,31)=34.08, M S E=0.782, p<$ $.001, \eta_{\mathrm{p}}^{2}=0.52$, but it was not for the foreshadow condition $(F$ $<1)$. This suggests that the most surprising event was an unforeshadowed shift. Ratings in this condition were the highest, whereas foreshadowing reduced the rating to the point where it was no longer significantly different from the no-shift conditions.

For causal stories, the shift comes about because the reason for the change in situation is unexplained. This means that the foreshadowing sentence should effectively act as the shift,

Table 3 Mean expectedness ratings in Experiment 1

\begin{tabular}{|c|c|c|c|c|}
\hline & \multicolumn{4}{|l|}{ Condition } \\
\hline & \multicolumn{2}{|c|}{ No foreshadow } & \multicolumn{2}{|l|}{ Foreshadow } \\
\hline & No shift & Shift & No shift & Shift \\
\hline Combined & $3.04(0.17)$ & $4.34(0.22)$ & $3.14(0.21)$ & $3.40(0.23)$ \\
\hline Casual & $2.92(0.32)$ & $5.25(0.36)$ & $3.34(0.42)$ & $3.43(0.32)$ \\
\hline
\end{tabular}

Note. Combined does not include causal stories. Standard errors appear in parentheses below the means. 
making the foreshadowed shift and foreshadowed no-shift conditions equivalent. Because of this, the data for these stories were separately submitted to a 2 (foreshadow) $\times 2$ (shift) ANOVA. While the main effect of foreshadow was not significant, $F(1,7)=2.60, M S E=1.493, p=.15, \eta_{\mathrm{p}}^{2}=$ 0.27 , the main effect of shift was, $F(1,7)=10.78, M S E=1.09$, $p=.013, \eta_{\mathrm{p}}^{2}=0.61$, as was the interaction, $F(1,7)=14.98$, $M S E=0.673, p=.006, \eta_{\mathrm{p}}^{2}=0.68$. Simple effects tests revealed that for the no-foreshadow condition, the effect of shift was significant $F(1,7)=28.14, M S E=0.774, p=.001, \eta_{\mathrm{p}}^{2}=0.80$, but it was not for the foreshadow condition $(F<1)$. The causal shift ratings mirror those reported for the other event shifts, again suggesting that the most surprising event is a shift that is not foreshadowed, while the other three conditions do not differ.

Overall, the pattern of results for Experiment 1 suggests that foreshadowing an event shift reduces the surprise with which readers react to that event. People rated the sentence containing the shift as less expected when it was not preceded by a sentence that indicated a possible change in the event. This result lends support to the idea that expectedness, and by extension, causal connectedness, plays a role in determining how a change in a situation model is processed. When a sentence foreshadows a potential change in a situation, people rate the shift as less surprising, potentially because they find it easier to causally connect the pre- and postshift information. The next question is whether these sentences, even when expected, are still recognized as conveying an event shift. This issue was explored in Experiment 2.

\section{Experiment 2}

The aim of Experiment 2 was to assess the degree to which foreshadowing affects the detection of event shifts. The question of interest was, Will readers still segment an event at a shift even if it is foreshadowed? Participants read stories and made responses indicating when a change in the event had occurred. This task is similar to those used in other studies of event segmentation, in which participants identify meaningful units of action (e.g., Kurby \& Zacks, 2008; Magliano, Kopp, McNerney, Radvansky, \& Zacks, 2012; Magliano, Miller, \& Zwaan, 2001; Newtson, 1973). Again, the shift was either foreshadowed or not.

\section{Method}

Participants One hundred twenty-eight people (69 female) were recruited through Amazon's Mechanical Turk website. Participants ranged in age from 18 to 62 years $(M=33.76, S D$ $=10.00$ ). Participants who failed to correctly answer 12 of 16 comprehension questions ( $75 \%$ ) were excluded. This resulted in the elimination of 23 participants. Participants were paid for their participation.

Materials and procedure The same materials in the same counterbalanced presentation order were used as in Experiment 1 . The procedure was also the same with the following modification: Instead of rating how expected each sentence was, participants indicated whether the situation had changed. Below each sentence, the question "Has the situation changed?" was displayed, and participants clicked a radio button next to a yes or no response. They were told that the definition of a change was up to them and that any change could give them the sense that the situation had changed in a meaningful way.

\section{Results and discussion}

As in Experiment 1, the proportion of subjects who identified shifts were collapsed across stories, and the analyses were done at the item level. The ratings for each condition are reported in Table 4 . The data were submitted to a 2 (foreshadow) $\times 2$ (shift) repeated-measures ANOVA.

For the noncausal stories, the main effect of foreshadow was not significant, $F(1,31)<1$, but the main effect of shift was, $F(1,31)=38.64, M S E=0.043, p<.001, \eta_{\mathrm{p}}^{2}=0.55$, so people identified shifts in all conditions. Importantly, the Foreshadow $\times$ Shift interaction was not significant, $F(1,31)$ $=1.65, M S E=0.038, p=.18, \eta_{\mathrm{p}}^{2}=.06$. Even when people had knowledge that a shift was likely to occur, they still recognized it by reading the sentence that conveyed it. The lack of interaction between foreshadow and shift suggests that the foreshadowing does not "erase" the shift, in comparison to Experiment 1 in which the foreshadowing did reduce the surprise associated with it.

Again, causal story data were analyzed separately because the foreshadowing sentence should act as a shift in those stories. These data were submitted to a 2 (foreshadow) $\times 2$ (shift) repeated-measures ANOVA. There were significant main effects of foreshadow, $F(1,7)=7.00, M S E=0.010, p$

Table 4 Proportion of subjects who identified a shift in Experiment 2

\begin{tabular}{llllll}
\hline & \multicolumn{3}{l}{ Condition } & & \\
\cline { 2 - 3 } & \multicolumn{2}{l}{ No foreshadow } & & \multicolumn{2}{l}{ Foreshadow } \\
\cline { 2 - 3 } \cline { 5 - 6 } & No shift & Shift & & No shift & Shift \\
\hline Combined & 0.288 & 0.562 & & 0.306 & 0.486 \\
& $(0.04)$ & $(0.04)$ & & $(0.05)$ & $(0.04)$ \\
Casual & 0.271 & 0.687 & & 0.238 & 0.530 \\
& $(0.06)$ & $(0.05)$ & & $(0.06)$ & $(0.09)$ \\
\hline
\end{tabular}

Note. Combined does not include casual stories. Standards errors appear in parentheses below the means. 
$=.03, \eta_{\mathrm{p}}^{2}=0.50$, and shift, $F(1,7)=16.70, M S E=0.060, p=$ $.005, \eta_{\mathrm{p}}^{2}=0.70$. The interaction was marginally significant, $F(1,7)=4.51, M S E=0.007, p=.07, \eta_{\mathrm{p}}^{2}=0.40$. Simple effects tests revealed a trend for a stronger shift effect in the noforeshadow condition than in the foreshadow condition, $F(1$, $7)=28.88, M S E=0.024, p=.001, \eta_{\mathrm{p}}^{2}=0.81$, and $F(1,7)=$ $7.96, M S E=0.043, p=.026, \eta_{\mathrm{p}}^{2}=0.53$, respectively, although the effect was clearly present in both. This result may be an artifact of the stories themselves. All of the shift sentences were written to be maximally obvious and disruptive to the reader. In causal shift sentences, the protagonist would react to the change in a sudden manner (e.g., one character reacts by cursing and jumping up and down; see Appendix), indicating a change in the situation. The abruptness of this behavior could still lead to the reader identifying a change in the situation, even after foreshadowing.

To more explicitly examine whether the foreshadow sentence was acting as a shift, a separate analysis of the proportion of subjects who identified shifts at foreshadow versus noforeshadow sentences was conducted. There was not a significant difference between the two sentence types, although there was a trend, $F(1,31)=2.88, M S E=0.033, p=.10, \eta_{\mathrm{p}}^{2}$ $=.09$. In the case of causal stories, in which the foreshadow sentence should act as a break, there was a significant difference, $F(1,7)=5.81, M S E=0.046, p=.047, \eta_{\mathrm{p}}^{2}=.45$, with more breaks identified at foreshadow sentences $(M=.59, S E=$ $.09)$ than at nonforeshadow sentences $(M=.33, S E=.07)$.

The pattern of results for Experiment 2 suggests that even when an event shift is foreshadowed, the change in the event, the event shift, is still recognized as such. This was true even for causal stories, where the foreshadowing could serve as a shift itself. This result, in conjunction with the result from Experiment 1, suggests that even when the amount of surprise, and potential causal disruption, associated with an event shift is reduced, people still recognize that a shift has occurred.

\section{Experiment 3}

The aim of Experiment 3 was to measure online processing of updating a situation model when an event shift was encountered, and the influence of foreshadowing on that processing. The results of Studies 1 and 2 and Experiment 1 suggest that people do not experience foreshadowed event shifts as surprising. If the previously observed reading-time increases are due to the cognitive effort required for situation model updating, then such increases should be observed, and be similar in magnitude, regardless of whether the event shift was foreshadowed. However, if the previously observed readingtime increase is due to readers' surprise at encountering an event shift, then there should be an attenuated or no increase in reading time if the event shift is foreshadowed.
Moreover, if foreshadowing attenuates a reading-time increase when event shifts are encountered, that may occur for two reasons. One possibility is that the foreshadowing is somehow disrupting of the processing of the event shift, and the situation model is not properly updated. If this is the case, then information that should be removed from a prior event should remain available after a shift. As such, people should respond to memory probes equally fast following a shift as not when it is foreshadowed, whereas when there is no foreshadowing, information from the prior event should be less available following a shift. Another possibility is that the foreshadowing causes the event shift to be more expected but that situation model updating still occurs. In this case, previously encountered information that is part of the prior event should be less available (Zwaan, 1996) regardless of whether there was foreshadowing of the event shift.

To test these two points, people in Experiment 3 read stories while their reading times were recorded and responded to critical probes that were presented after shift sentences.

\section{Method}

Participants Forty-eight people (23 female) were recruited through Amazon's Mechanical Turk website. ${ }^{2}$ Participants ranged in age from 19 to 68 years $(M=37.39, S D=11.39)$. Participants who failed to correctly answer 30 of 40 comprehension questions $(75 \%)$ were excluded. This resulted in the elimination of 16 participants; one additional participant was replaced for having low probe accuracy $(6.25 \%$ correct). Participants were paid for their participation.

Materials and procedure The same materials were used as in Experiments 1 and 2. The procedure was also the same with the following modifications: Participants read a total of 40 stories, eight in each of four shift conditions (causal stories were not included) plus eight filler stories. The stories were modified such that they displayed one or two probes centered on a separate screen per story in all capital letters. The critical probes were all verbs that appeared in the text and described actions that would no longer be occurring after a shift. For example one story described a character listening to a conversation. Following a spatial shift, this would no longer be possible. Filler probes were verbs that did not appear in the story. Critical probes always appeared immediately following the shift or no-shift sentence. Filler probes appeared elsewhere in the story with the limitation that they never came between a foreshadow/no-foreshadow sentence and a shift/no-shift sentence. Responses were made by pressing the $g$ button on

\footnotetext{
${ }^{2}$ Although collecting reading and response times online is less than ideal, the authors believe that there is merit to this method of data collection. Steps were taken to reduce the impact of potential limitations ,including eliminating responses from people who used a phone/tablet and only including data from those who were using a broadband connection.
} 
the keyboard to indicate the probe word had been seen before or the $h$ button to indicate it was new. Participants did not rate the expectedness of the sentences, nor did they explicitly indicate the presence of event shifts.

\section{Results and discussion}

Reading times for Experiment 3 are presented in Table 5. For the reading-time data, any reading times less than $50 \mathrm{~ms} /$ syllable or greater than $500 \mathrm{~ms} / \mathrm{syllable}$ were trimmed. This resulted in the elimination of 106 reading times, or $10.35 \%$ of the data. The remaining times were averaged; no additional reading times were trimmed for being greater than the average plus three standard deviations.

The reading time data were submitted to a 2 (foreshadow) $\times 2$ (shift) repeated-measures ANOVA. For Experiment 3, because all participants were exposed to each condition, analysis was done at the participant level. The main effect of foreshadowing was marginally significant, $F(1,31)=4.00, M S E=549, p=$ $.054, \eta_{\mathrm{p}}^{2}=0.11$, and the main effect of shift was not, $F(1,31)=$ $1.85, p=.18$. This was qualified by a significant interaction, $F(1$, $31)=5.27, M S E=356, p=.029, \eta_{\mathrm{p}}^{2}=0.15$. Simple effects tests revealed that when an event shift was foreshadowed, there was no difference in reading times between the shift and no-shift conditions $(F<1)$. However, in the no-foreshadow condition, there was a difference, $F(1,31)=6.39, M S E=450, p=.017, \eta_{\mathrm{p}}^{2}$ $=0.17$, with longer reading times in the shift condition. ${ }^{3}$ Thus, providing information about an upcoming event shift reduced the processing disruption that occurred when a reader encountered that shift. This result leads to the question addressed by the second aim of Experiment 3. Even though people recognize event shifts in the presence of foreshadowing, as shown in Experiment 2, how does foreshadowing affect their mental representations of the events involved?

To address this, probe response times and accuracy rates were analyzed. Response times and accuracy rates are presented in Table 5. These data were submitted to separate 2 (foreshadow) $\times 2$ (shift) repeated measures ANOVAs. For the response times, there was a significant main effect of shift, $F(1,31)=7.09, M S E=150,313, p=.012, \eta_{\mathrm{p}}^{2}=0.19$, with response times slower in the shift condition. Neither the main effect of foreshadow nor the interaction were significant $\left(F_{\mathrm{s}}<\right.$ 1). For accuracy rates, neither the main effect of foreshadow nor shift (both $F_{\mathrm{s}}<1.23$ ), nor the interaction were significant $(F<1)$. These results are consistent with Zwaan (1996), who found that response latencies to recognition probes were slower following temporal shifts but that error rates were

\footnotetext{
${ }^{3}$ An additional reading time study without the probe task produced the same results. That is, there was a significant Foreshadow $\times$ Shift interaction with slower reading times following a shift in the no-foreshadow condition only. Thus, these reading time patterns are not a result of using a memory probe.
}

Table 5 Reading times, probe response times, and accuracy rates in Experiment 3

\begin{tabular}{llllll}
\hline & \multicolumn{2}{l}{ No foreshadow } & & \multicolumn{2}{l}{ Foreshadow } \\
\cline { 2 - 3 } \cline { 5 - 6 } & No shift & Shift & & No shift & Shift \\
\hline $\begin{array}{c}\text { Reading time/ } \\
\text { syllable (ms) }\end{array}$ & $149(14)$ & $162(14)$ & $148(112)$ & $147(12)$ \\
$\begin{array}{c}\text { Response } \\
\text { time (ms) }\end{array}$ & $2,853(120)$ & $3,012(142)$ & $2,894(166)$ & $3,099(148)$ \\
Accuracy & $0.836(0.03)$ & $0.801(0.03)$ & $0.801(0.03)$ & $0.781(0.03)$ \\
\hline
\end{tabular}

Note. Standard errors appear in parentheses below means.

generally not different. Thus, readers were updating their event models, as response times were slower following an event shift, even when that event shift was foreshadowed.

The results of Experiment 3 show that reading times are influenced by foreshadowing. When an event shift was foreshadowed, reading times were not as disrupted as they were when the shift was not foreshadowed. Foreshadowing removes the causal break that may occur when a person encounters a shift while reading. Moreover, information from a prior event was less available, regardless of foreshadow condition. Thus, even though an event shift may be easier to process after foreshadowing, the new event still enjoys a privileged status in memory while the previous model becomes less available, consistent with other work.

\section{General discussion}

The results of two studies using previous materials from Radvansky et al. (2001) and three experiments using materials designed for this study demonstrate that during reading comprehension, when people are reading multiple, experimentally constructed texts, the presence of an event shift may be unexpected, and that this, at least in part, is producing the readingtime increase that has been repeatedly reported in the literature. The results of Studies 1 and 2 show that the event shifts in previously used texts are unexpected and that these levels of expectedness can influence reading times, diminishing the influence of the event shifts per se. Experiment 1 shows that knowing that a shift is likely to occur when it has been foreshadowed in the text changes the reader's perception of that shift. What might, by itself, be surprising to the reader becomes less surprising with foreshadowing.

One possible explanation of the results of Experiment 1 is that the foreshadowing sentence itself acts as a shift. That is, the event shift is just being moved to earlier in the text. If this were the case, then the shift sentence should no longer be recognized as a shift because the foreshadowing sentence would be filling this role. However, the results of Experiment 2 show that, even when the shift was 
foreshadowed, participants still identified a change in the situation at the shift-conveying sentence at the same rate as when the foreshadowing did not occur. Even for causal shifts, where the foreshadowing is expected to replace the shift, people responded that there was a change in the described eventsan event shift. Although, this may be the result of an additional change in the situation brought about by the characters' strong reactions to the event shift. Additionally, analysis of the foreshadow sentences did not find a significant difference in the proportion of people who identified a shift in the foreshadow versus no-foreshadow conditions. Thus, it does not appear that people are simply moving the shift to the previous sentence.

Experiment 3 examined the influence of event shift foreshadowing on measures of online processing — namely, reading time and probe response time. For the reading-time data, shift sentences were not read slower when the shift was foreshadowed, indicating that people were not as disrupted in their comprehension of the stories as they otherwise might have been. This supports the idea that foreshadowing prior to an event shift can reduce the surprise readers may experience when they encounter an event shift in a text. At the same time, information was less available following an event shift, even when that shift was foreshadowed. This suggests that people still updated their situation models when they encountered the event shift.

One implication of the result of Experiment 3 is that when it is easier to link two different events, less mental processing (as measured by reading time) is required to account for the change. This might seem to contradict EST, as EST says that the when the discrepancy between what is expected and what actually happens becomes too large, updating occurs. However, expecting that a situation will change and being able to successfully predict what that change will be are very different things. The foreshadow sentence prompted people to expect a change, reducing the amount of surprise associated with the event boundary. However, these sentences did not have predictive value, so they should not change the amount of discrepancy between the old and new situations. Thus, updating still occurs.

Overall, the data support the idea that expectedness plays a role in influencing the way event shifts are processed during reading comprehension. The finding that people read foreshadowed shift sentences faster than nonforeshadowed shift sentences suggests that the reading-time increase that has been observed in prior work is due, at least in part, to the unexpectedness of the event shift itself. It may also be that less mental effort is required to incorporate the event shift when information encountered at a shift can be causally connected to preshift information. While the reading times remained unaffected following a foreshadowed shift, the shift itself still appears to be fully processed because information from the no-longer-relevant situation model is not as available as it otherwise would be.

The results presented here dovetail nicely with another series of experiments which found that predictability was related to event segmentation and reading time (Zacks, Speer, \&
Reynolds, 2009). In one experiment, participants rated the predictability of a series of clauses from a book; clauses that contained one or more changes were rated as less predictable. Furthermore, predictability ratings were related to both identification of event shifts and increases in reading time (Zacks et al., 2009). There are a couple of important differences in the present results and those of Zacks et al. First, they found that causal changes were associated with reduced predictability ratings, whereas changes in the other model variables discussed above (plus changes in object) were either not significant or, in the case of entity changes, were associated with increased predictability. However, as was the case in some of the previously discussed research (e.g., McNerney et al., 2011; Radvansky et al., 2001), which sometimes found decreases in reading time at event shifts, the materials used in Zacks et al. were more naturalistic, as they came from a book, and clauses could contain multiple shifts. In these cases, with more information available, it may be that people are better able to predict what will happen next and can immediately adjust to that change. Alternatively, because the more naturalistic materials provide a rich source of detail, it may allow readers to disregard certain changes as the reason for the change should become apparent. For example, in a novel, such as that used by McNerney et al. (2011), changes in space are likely to be explained in the sentences that immediately follow. This expectation could allow readers to devote less time to adjust to the change, so reading times for the shift-conveying sentence would be unaffected.

Another difference is that in a hierarchical regression, the relationship between predictability and reading time was reduced by adding structural variables (e.g., punctuation, clause length) to the model and was not affected when situation model variables were added. As Zacks et al. (2009) point out in their discussion, the structural variables in their materials were correlated with situation changes, which would attenuate the effect of these changes. Overall, the results lend support to the idea that causality and expectedness are linked in the identification of event changes and that, even in more realistic texts, causal relations are key to the reader's understanding of the narrative.

Future research in this area could address the effect of ease of making causal connections on later memory for information contained in different situation models. For example, it may be that when the cause of a shift is easily connected to the current model, it is easier to incorporate the new information, and there would be better recall of previously encountered information than there otherwise would be following some delay. Previous research has found that people remember more information from texts that contain a shift than those that do not (Pettijohn, Thompson, Tamplin, Krawietz, \& Radvansky, 2016). Reducing the amount of effort needed to process a shift may impact the amount of information subsequently remembered. That said, the additional time and effort needed to process a shift may make that information more memorable, specifically the information immediately surrounding the shift (e.g., Swallow, Zacks, \& 
Abrams, 2009). Understanding these issues could aid the creation of training or educational materials by designing the text in such a way that the ultimate retention of information is maximized.
The next morning she got up and turned on the radio. (Shift)

The morning crew was talking about traffic, so she switched it off.

\section{Appendix}

\section{Example Temporal Shift Story}

No Foreshadow No Shift

Jenny was listening to the radio.

She had been stressed all day and was finally unwinding.

The DJ was spinning some really great stuff.

She really liked this song, especially the pounding bass line. (No Foreshadow)

She turned up the volume and adjusted the bass level. (No Shift)

It was loud enough to rattle her windows.

No Foreshadow Shift

Jenny was listening to the radio.

She had been stressed all day and was finally unwinding.

The DJ was spinning some really great stuff.

She really liked this song, especially the pounding bass line. (No Foreshadow)

The next morning she got up and turned on the radio. (Shift)

The morning crew was talking about traffic, so she switched it off.

\section{Foreshadow No Shift}

Jenny was listening to the radio.

She had been stressed all day and was finally unwinding.

The DJ was spinning some really great stuff.

Unfortunately, she would have to call it a night soon. (Foreshadow)

She turned up the volume and adjusted the bass level. (No Shift)

It was loud enough to rattle her windows.

Foreshadow Shift

Jenny was listening to the radio.

She had been stressed all day and was finally unwinding.

The DJ was spinning some really great stuff.

Unfortunately, she would have to call it a night soon. (Foreshadow)

\section{Example Causal Story}

No Foreshadow No Shift

Allan was on a hot streak bowling tonight.

It was only the fifth frame, and he already had four strikes and a spare.

He could see that it was going to be a good night. (No Foreshadow)

He might even beat his personal best. (No Shift)

The guy next to him had slipped and rolled his ball into Allan's ankle.

Allan realized the night might not be so great after all.

No Foreshadow Shift

Allan was on a hot streak bowling tonight.

It was only the fifth frame, and he already had four strikes and a spare.

He could see that it was going to be a good night. (No Foreshadow)

He began to curse and jump up and down. (Shift)

The guy next to him had slipped and rolled his ball into Allan's ankle.

Allan realized the night might not be so great after all.

Foreshadow No Shift

Allan was on a hot streak bowling tonight.

It was only the fifth frame, and he already had four strikes and a spare.

He saw a ball from the next lane coming toward him. (Foreshadow)

He might even beat his personal best. (No Shift)

The guy next to him had slipped and rolled his ball into Allan's ankle.

Allan realized the night might not be so great after all.

Foreshadow Shift

Allan was on a hot streak bowling tonight.

It was only the fifth frame, and he already had four strikes and a spare.

He saw a ball from the next lane coming toward him. (Foreshadow)

He began to curse and jump up and down. (Shift)

The guy next to him had slipped and rolled his ball into Allan's ankle.

Allan realized the night might not be so great after all. 


\section{References}

Albrecht, J. E., \& O'Brien, E. J. (1993). Updating a mental model: Maintaining both local and global coherence. Journal of Experimental Psychology: Learning, Memory, \& Cognition, 19(5), 1061-1070.

Anderson, A., Garrod, S. C., \& Sanford, A. J. (1983). The accessibility of pronominal antecedents as a function of episode shifts in narrative texts. Quarterly Journal of Experimental Psychology, 35(A), 427440.

Bates, D., Mächler, M., Bolker, B., \& Walker, S. (2015). Fitting linear mixed-effects models using lme4. Journal of Statistical Software, $67(1), 1-48$.

Glenberg, A. M., Meyer, M., \& Lindem, K. (1987). Mental models contribute to foregrounding during text comprehension. Journal of Memory and Language, 26(1), 69-83. doi:10.1016/0749596X(87)90063-5

Graesser, A. C., Singer, M., \& Trabasso, T. (1994). Constructing inferences during narrative text comprehension. Psychological Review, 101(3), 371-395.

Jahn, G. (2004). Three turtles in danger: Spontaneous construction of causally relevant spatial situation models. Journal of Experimental Psychology: Learning, Memory, and Cognition, 30(5), 969-987. doi:10.1037/0278-7393.30.5.969

Johnson-Laird, P. N. (1983). Mental models. Cambridge, MA: Harvard University Press.

Kurby, C. A., \& Zacks, J. M. (2008). Segmentation in the perception and memory of events. Trends in Cognitive Sciences, 12(2), 72-79.

Kurby, C. A., \& Zacks, J. M. (2012). Starting from scratch and building brick by brick in comprehension. Memory \& Cognition, 40(5), 812826.

Kuznetsova, A., Brockhoff, P. B., \& Christensen, R. H. B. (2013). lmerTest: Tests for random and fixed effects for linear fixed effects models (lmer objects of lme4 package). Retrieved from http:// CRAN.R-project.org $/$ package $=$ lmerTest

Lea, R. B., Kayser, P. A., Mulligan, E. J., \& Myers, J. L. (2002). Do readers make inferences about conversational topics? Memory \& Cognition, 30(6), 945-957.

Long, D. L., \& Lea, R. B. (2005). Have we been searching for meaning in all the wrong places? Defining the "search after meaning" principle in comprehension. Discourse Processes, 39(2/3), 279-298.

Magliano, J., Kopp, K., McNerney, M. W., Radvansky, G. A., \& Zacks, J. M. (2012). Aging and perceived event structure as a function of modality. Aging, Neuropsychology, and Cognition, 19(1/2), 264 282

Magliano, J. P., Miller, J., \& Zwaan, R. A. (2001). Indexing space and time in film understanding. Applied Cognitive Psychology, 15(5), $533-545$.

McNerney, M. W., Goodwin, K. A., \& Radvansky, G. A. (2011). A novel study: A situation model analysis of reading times. Discourse Processes, 48, 453-474.

Newtson, D. (1973). Attribution and the unit of perception of ongoing behavior. Journal of Personality and Social Psychology, 28(1), 2838.

O'Brien, E. J., \& Albrecht, J. E. (1991). The role of context in accessing antecedents in text. Journal of Experimental Psychology: Learning, Memory, \& Cognition, 17(1), 94-102.
Pettijohn, K. A., Thompson, A. N., Tamplin, A. K., Krawietz, S. A., \& Radvansky, G. A. (2016). Event boundaries and memory improvement. Cognition, 148, 136-144.

R Core Team. (2013). R: A language and environment for statistical computing [Computer software]. Vienna, Austria: R Foundation for Statistical Computing. Retrieved from http://www.R-project.org/

Radvansky, G. A., \& Copeland, D. E. (2000). Functionality and spatial relations in memory and language. Memory \& Cognition, 28(6), 987-992.

Radvansky, G. A., \& Copeland, D. E. (2010). Reading times and the detection of event shift processing. Journal of Experimental Psychology: Learning, Memory, \& Cognition, 36(1), 210-216.

Radvansky, G. A., Copeland, D. E., \& Zwaan, R. A. (2003). Aging and functional spatial relations in comprehension and memory. Psychology and Aging, 18(1), 161-165.

Radvansky, G. A., Zwaan, R. A., Curiel, J. M., \& Copeland, D. E. (2001). Situation models and aging. Psychology and Aging, 16(1), 145-160.

Rapp, D. N., \& Taylor, H. A. (2004). Interactive dimensions in the construction of mental representations for text. Journal of Experimental Psychology: Learning, Memory, and Cognition, 30(5), 988-1001.

Rinck, M., \& Weber, U. (2003). Who when where: An experimental test of the event-indexing model. Memory \& Cognition, 31(8), 1284 1292.

Speer, N. K., Zacks, J. M., \& Reynolds, J. R. (2007). Human brain activity time-locked to narrative event boundaries. Psychological Science, 18(5), 449-455.

Sundermeier, B. A., van den Broek, P., \& Zwaan, R. A. (2005). Causal coherence and the availability of locations and objects during narrative comprehension. Memory \& Cognition, 33(3), 462-470.

Swallow, K. M., Zacks, J. M., \& Abrams, R. A. (2009). Event boundaries in perception affect memory encoding and updating. Journal of Experimental Psychology: General, 138(2), 236-257.

van Dijk, T. A., \& Kintsch, W. (1983). Strategies in discourse comprehension. New York, NY: Academic Press.

Zacks, J. M., Speer, N. K., Swallow, K. M., Braver, T. S., \& Reynolds, J. R. (2007). Event perception: A mind-brain perspective. Psychological Bulletin, 133(2), 273-293.

Zacks, J. M., Speer, N. K., \& Reynolds, J. R. (2009). Segmentation in reading and film comprehension. Journal of Experimental Psychology: General, 138(2), 307-327.

Zwaan, R. A. (1996). Processing narrative time shifts. Journal of Experimental Psychology: Learning, Memory, and Cognition, 22(5), 1196-1207.

Zwaan, R. A., Langston, M. C., \& Graesser, A. C. (1995). The construction of situation models in narrative comprehension: An eventindexing model. Psychological Science, 6(5), 292-297.

Zwaan, R. A., Magliano, J. P., \& Graesser, A. C. (1995). Dimensions of situation model construction in narrative comprehension. Journal of Experimental Psychology: Learning, Memory, and Cognition, 21(2), 386-397.

Zwaan, R. A., \& Radvansky, G. A. (1998). Situation models in language comprehension and memory. Psychological Bulletin, 123(2), 162185.

Zwaan, R. A., Radvansky, G. A., Hilliard, A. E., \& Curiel, J. M. (1998). Constructing multidimensional situation models during reading. Scientific Studies of Reading, 2(3), 199-220. 